\title{
Clinical leadership development and education for nurses: prospects and opportunities
}

This article was published in the following Dove Press journal:

Journal of Healthcare Leadership

22 July 2015

Number of times this article has been viewed

\author{
M Lindell Joseph \\ Diane L Huber \\ College of Nursing, The University \\ of lowa, lowa City, IA, USA
}

Correspondence: $M$ Lindell Joseph College of Nursing, The University of lowa, 50 Newton Road, Room Number 466 CNB, lowa City, IA 52242, USA Tel + I 3193353609

Email maria-joseph@uiowa.edu

\begin{abstract}
With the implementation of the Affordable Care Act, elevated roles for nurses of care coordinator, clinical nurse leader, and advanced practice registered nurse have come to the forefront. Because change occurs so fast, matching development and education to job requirements is a challenging forecasting endeavor. The purpose of this article is to envision clinical leadership development and education opportunities for three emerging roles. The adoption of a common framework for intentional leadership development is proposed for clinical leadership development across the continuum of care. Solutions of innovation and interdependency are framed as core concepts that serve as an opportunity to better inform clinical leadership development and education. Additionally, strategies are proposed to advance knowledge, skills, and abilities for crucial implementation of improvements and new solutions at the point of care.

Keywords: clinical leadership, nursing leadership, CNL, care coordination, innovation, interdependency
\end{abstract}

\section{Clinical leadership development and education for nurses: prospects and opportunities}

Complexity, chaos, high rates of change, serious safety and quality issues, and workforce shortages in health care are some of the reasons why clinical leadership is important. Although the future may be difficult to predict exactly, history has taught us that it is crucial to prepare nurses for key roles in the health care delivery system, both for present jobs and for potential future opportunities. The challenge for clinical leadership is how to structure the knowledge, skills, and abilities required so that nurses are positioned to step into roles that are needed, yet, for which there may not yet be formal jobs. One example is expanding and elevating the registered nurse $(\mathrm{RN})$ role from traditional care delivery to integrating care, where they are working with autonomy, authority, and accountability in managing and coordinating care across disciplines and settings. ${ }^{1}$ Smolowitz et al ${ }^{2}$ studied 16 primary health care practices that used RNs to the full extent of their scope of practice in team-based care and found that episodic and preventive care, chronic disease management, and practice operations were the three main aspects of RN activities. They noted that there is a compelling need to expand the contributions and optimize the scope of practice of RNs in primary health care for leadership in interprofessional teams. The purpose of this article is to envision clinical leadership opportunities for three nursing roles: specifically, care coordinator, clinical nurse leader (CNL), and advanced practice RN (APRN), and discuss a common framework for leadership development and education. 


\section{The changing face of health care}

The Patient Protection and Affordable Care Act (ACA) was passed and signed into law in 2010. Subsequent implementation has contributed to reconfiguration in health care delivery, accelerated the demand for health care along with a shortage of key health care professionals, and opened up new and expanded roles for nurses under new care delivery models. Aimed at extending health insurance coverage, there are many provisions of the ACA, including those designed to emphasize prevention and wellness, improve quality and system performance, and curb costs. Notable among these aspects are the creation of health homes and integration of care for persons with chronic illnesses, improvements in care coordination, emphasis on prevention and primary care, investment in health information technology, and testing of new delivery and payment systems. Authorized under the ACA for Medicare reimbursement, the Centers for Medicare and Medicaid Services (CMS) has implemented an initiative to reward acute care hospitals with quality-of-care incentive payments for the quality of care delivered to Medicare patients. Called Hospital Value-Based Purchasing (VBP), this program bases payment (or nonpayment) to hospitals on how closely they follow best clinical practices and how well they enhance patients' experience of care. The goal is to link payment to a value-based system that improves quality of care and is not just based on volume of services.

These changes in the context of health care delivery have driven the need to supercharge RNs' leadership knowledge, skills, and abilities and develop targeted care coordination and integration competencies for a more robust skill set that is matched to changing clinical leadership imperatives. Because of ACA and VBP, there is an urgent need for innovation and better management of reciprocal interdependence. Nurses hold the central ground for quality, safety, and improving the patient experience. For example, having quick care clinics affiliated with a hospital can decrease nonemergent patients seeking health care on weekends and nights in the emergency department and increase patient satisfaction with not having to wait. However, to run smoothly, nurse clinical leadership is needed to coordinate and integrate care with affiliated sites such as laboratories, imaging centers, and pharmacies.

\section{What is clinical leadership in nursing?}

Leadership in nursing is highly valued. The Institute of Medicine $^{3}$ noted that nurses need to be prepared to lead in all aspects of health care. Leadership is defined as "the process of influencing people to accomplish goals. Key concepts related to leadership are influence, communication, group process, goal attainment, and motivation". ${ }^{4}$ In order to understand clinical leadership, it needs to be defined. Clinical leadership is defined here as the process of influencing pointof-care innovation and improvement in both organizational processes and individual care practices to achieve quality and safety of care outcomes. McCausland ${ }^{5}$ noted that new interdisciplinary models of care that cross traditional boundaries of ambulatory, inpatient, and community settings need credible clinical leaders. Thomas and Roussel ${ }^{6}$ noted that clinical leadership is about clinicians augmenting care for safety and quality by using innovation and improvement. This places the opportunity for health care leadership at the clinical level within the realm of each direct provider of care, especially nurses who are at the direct care level. They stated "There is a need for a more programmatic, strategic approach to clinical leadership, because the United States' ailing health care system is in urgent need of reform". ${ }^{6}$ Thus, clinical leadership needs targeted preparation.

Clinical leadership uses the skills of the RN and adds components of general leadership skills, skills in management of care delivery at the point of care, and focused skills in using evidence-based practice for problem solving and outcomes management. There is clearly a need for clinical leadership in nursing because of the many and varied point-of-care implementation problems that arise. For example, patient safety may be compromised if there is poor team communication. When nurses are busy or short-staffed, hand-off communications may be compromised, creating gaps in care (Huber et al, unpublished data, 2015). Discharge transitions may not be smooth if both sending and receiving areas do not communicate well. Medication administration may be less than smooth when multiple disciplines caring for a patient do not coordinate prescribing and transcribing practices. This is true in acute care and across the continuum. Resolving ongoing care gaps requires energetic actions based on best practices, teamwork, care coordination, and clinical leadership competencies at the point of care.

\section{Evidence base for clinical leadership}

There is a body of evidence demonstrating the relationship between nursing leadership and patient outcomes. ${ }^{7}$ Wong et al ${ }^{7}$ found 19 patient outcome variables were related to leadership in the 5 categories of patient satisfaction: patient mortality, patient safety outcomes, adverse events, complications, and patient health care use. Their systematic review of 20 studies from 2005 to 2012 on the relationship of nursing leadership practices and patient outcomes demonstrated a positive relationship between relational leadership and multiple patient 
outcomes. A connection between supportive leadership styles and positive patient safety outcomes was noted. They concluded that "the current evidence suggests a clear relationship between relational leadership styles and lower patient mortality and reduced medication errors, restraint use, and hospital-acquired infections". ${ }^{7}$ Relational leadership styles were also positively and indirectly related to improved work environments and outcomes for nurses. ${ }^{8}$ This suggests that it is important for leaders to understand the patient care processes and the role of nurses in promoting better outcomes. Among the 20 studies reviewed, transformational leadership was the most frequently used leadership theory.

Transformational leadership is an evidence-based theory used as a strategy and manifested as a style for working within the complexity of care and the use of interdisciplinary teams. ${ }^{9}$ It is a relational leadership style and an evidence-based management practice that can form the basis of practical clinical leadership development and education. For example, the American Organization of Nurse Executives (AONE) ${ }^{10}$ has identified five core nurse executive competencies: leadership, communication and relationship-building, business skills, knowledge of the health care environment, and professionalism. Transformational leadership directly addresses some of the competencies in the first category of leadership. There is a solid body of evidence that transformational leadership is related to effectiveness, ${ }^{9-12}$ which is especially important for working with interprofessional teams, managing the coordination of care, and innovating roles and functions as structures are changing.

\section{Clinical leadership roles}

Clinical leadership roles are often thought of as targeted to the development of nurse managers and executives. Given the need for clinical leadership development at all levels, the focus here is on the development and education of nurses as leaders who are prepared to lead at the unit, program, or microsystem level and across the continuum of care. This is the "cutting edge" where the operations of care delivery occur. Nurses in direct care roles deliver care to and coordinate the care of patients and clients. Yet, there are organizational and systems imperatives for quality and safety initiatives and innovations designed and executed by nurses. Thus, to fully enact the direct care role, nurses must be prepared to address all situations that arise at the intersection of clinical practice (provider with patient and family) with the context and environment of care (organizations and groups of multiple care providers and disciplines). This is the imperative of clinical leadership. For example, the leadership and management of care transitions, both within and across settings and sites of care, is a crucial function under implementation of the ACA and its VBP financial aspects. The traditional preparation of nurses has not emphasized these roles and functions, but rather mastery of the psychomotor and conceptual skills needed to deliver entry-level care as an RN. The US health care system has been predominantly acute care driven. Thus, there has been heavy emphasis on learning directed to acute care practice and disease-specific knowledge rather than management of populations, chronic conditions management, care integration, and care coordination among multiple disciplines and in multidisciplinary team care delivery models. A near-term acceleration of care delivery reconfigured to outpatient and primary care will change nurses' practice settings and job requirements, including expanding roles and levels of autonomy. These changes will impact nurses' development and educational needs. In studying new and elevated roles for nurses as care integrators, Joynt and Kimball ${ }^{1}$ identified the following examples: serving as team leaders, CNLs serving as unit-based care managers, nurse practitioners serving as primary care providers in clinics, and nurse coaches managing transitions across settings. Three major clinical leadership roles for nurses that are positioned to enable the expectations of the ACA are care coordinator, CNL, and APRN. Since these emerging roles support the ACA, we will discuss the preparation and developmental opportunities for these roles.

\section{Care coordinator role}

As the health care environment has been changing and care is shifting to population management and outpatient settings, the care coordinator role has emerged as a new twist on case management and a new model of professional nursing practice. Nurses are often responsible for coordinating care for a group or population of patients. ${ }^{13}$ However, this has been housed within the context of acute care delivery in the past. For example, nurses may manage populations of patients with diabetes or cardiovascular disease in acute care. In the new and emerging models, nurses are and will be managing many types of carved out populations with chronic illnesses or behavioral health conditions across settings and sites and for long time frames. New roles and jobs have been the natural result. Contemporary names are care coordinators, health coaches, navigators, or care managers. These roles have arisen in conjunction with shifts to patient-centered medical homes and accountable care organizations under the ACA and reimbursement shifts that have put renewed emphasis on care coordination, care management, and prevention strategies 
related to VBP aspects. ${ }^{14}$ Care coordinators often serve as case managers, and care coordination is at the core of case management practice.

Case managers have a long and distinguished history of service delivery in nursing and social work. Education for the care coordinator role arises from education within the discipline (eg, nursing or social work) and often includes specialty knowledge and experience in case or population health management. There is no generally acknowledged curriculum for education and training of care coordinators or case managers, but there is a text that is a core curriculum for addressing the case manager certification exam. ${ }^{15}$ At this point, development and education for nurses in care coordination roles tend to be outside of formal graduate education. The professional organization, the Case Management Society of America (CMSA), is the major body representing case managers. It issues standards of practice and links with transitions of care organizations. CMSA's annual conference is a key educational event.

Case managers practice within a variety of professional disciplines. The top two work settings for case managers are health plans $(28.8 \%)$ and hospitals $(22.8 \%)$; most case managers are RNs $(88.6 \%) .{ }^{16}$ Certification is not universally mandatory; however, about $40 \%$ of employers require board certification. ${ }^{16}$ There are many certifications available. The Commission for Case Manager Certification (CCMC) is the oldest, largest, and most recognized of the certifications. They use field-tested role and function studies as the basis of their certification, qualifications, and test plan. CCMC has identified eight essential activities of case management: assessment, planning, implementation, coordination, monitoring, evaluation, outcomes, and general aspects. The six core components of case management are: 1) psychosocial aspects; 2) health care reimbursement; 3) rehabilitation; 4) health care management and delivery; 5) principles of practice; and 6) case management concepts. ${ }^{17}$ These six domains form the knowledge content areas for the exam's test plan. They can be used to guide studying for the exam. In addition, CCMC offers many other resources for case management practice, such as a code of ethics called the code of professional conduct. The California Institute for Nursing and Healthcare $(\mathrm{CINH})$ conducted a nurse role exploration project. These authors call for new settings and contexts for experiential learning activities for care coordinators to enable collaboration and skill development across the continuum of care versus traditional settings and approaches. ${ }^{18}$ That being said, intentional leadership development will be required for current and future care coordinators roles (Figure 1).

\section{Clinical nurse leader}

Specific to nursing, the CNL role was conceptualized and developed by the American Association of Colleges of Nursing (AACN) and nursing leaders in 2003. ${ }^{19}$ It was a new role in nursing, with preparation as an advanced generalist focused on transforming care at the unit of service or point of care. ${ }^{20}$ The CNL role is especially suited to collaborative

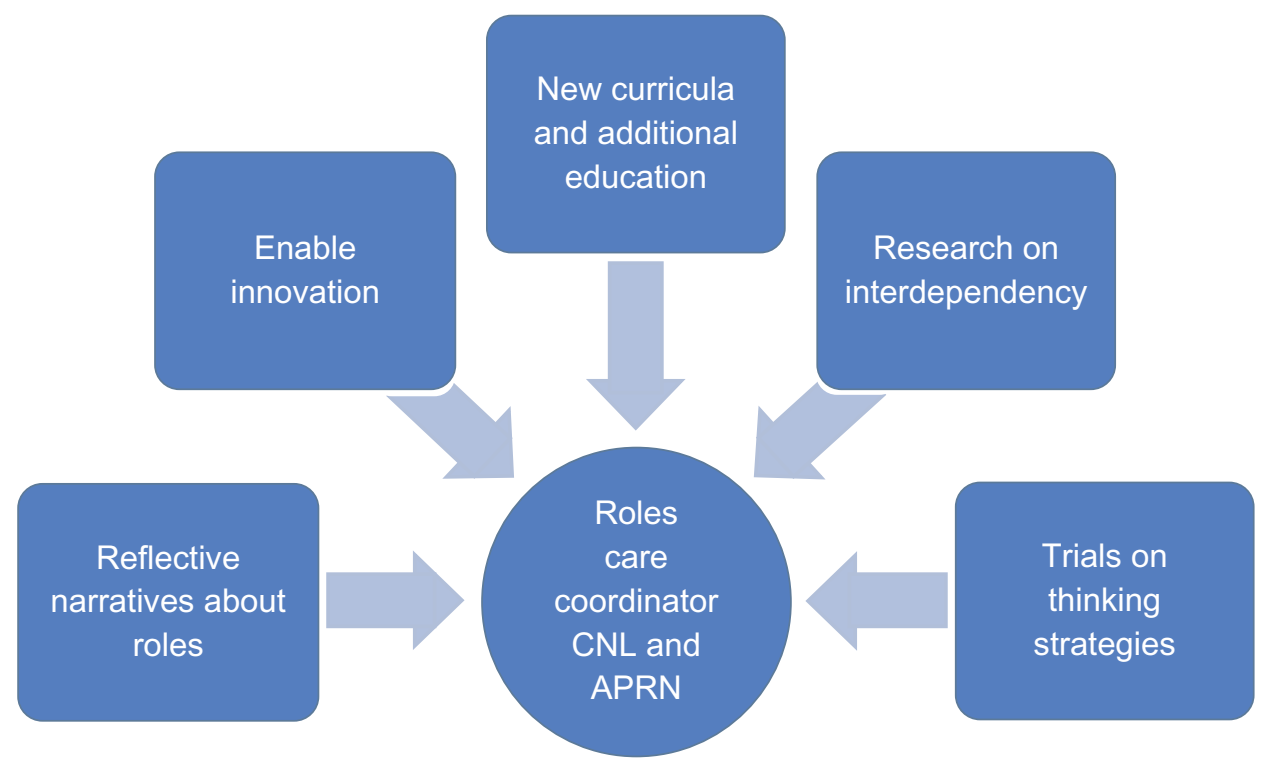

Figure I Framework for intentional clinical leadership development.

Abbreviations: CNL, clinical nurse leader; APRN, advanced practice registered nurse. 
work with interprofessional teams and in the coordination of care. Prepared at the Master's level, CNLs acquire the knowledge, skills, and abilities for evidence-based practice, care coordination, teamwork, quality and safety, outcomes management, and operational management of a complex microsystem. The CNL has been described as a front-line innovator. One of the ultimate aims of the CNL is to improve patient care outcomes, costs, and satisfaction in the microsystem through the development of eight competencies, which include those of clinician, educator, advocate, outcomes manager, information manager, team manager, system analyst/risk anticipation, and member of the profession.

CNL education helps prepare nurses for opportunities to make improvements in systems at the point of care, where changes closely impact patients and families. The CNL curriculum framework centers on the domains of nursing leadership, clinical outcomes management, and care environment management. ${ }^{19}$ AACN has promulgated the core curriculum essentials for the CNL. ${ }^{21}$ Under the nursing leadership domain, major areas of emphasis are horizontal leadership, effective use of self, advocacy, the CNL role, and lateral integration of care. Under the clinical outcomes management domain, major areas of emphasis are illness/disease management, knowledge management, health promotion and disease, and evidencebased practice. Under the care environment management domain, major areas of emphasis are team coordination, health care finance/economics, health care systems and organizations, health care policy, quality management/ risk reduction/patient safety, and informatics. This CNL curriculum framework outlines the needed development and education to be integrated with ten threads of critical thinking, communication, ethics, human diversity/cultural competence, global health care, professional development, accountability, assessment, nursing technology, and professional values in both didactic and clinical experiences within the educational program. ${ }^{19}$ The formal program of study for the CNL culminates with a certification exam administered by AACN. CNL education prepares nurses to focus on transforming care at the point of care. CNLs have advanced knowledge, skills, and abilities in quality improvement, outcomes measurement and management, systems management, and changing leadership to bring to bear on transforming care. According to Binder, ${ }^{22}$ little is known about structures and processes that influence successful integration, and components that influence or hinder effectiveness and sustainability of the CNL role. Therefore, the intentional framework serves to provide enabling education to influence role effectiveness (Figure 1).

\section{Advanced practice registered nurses}

APRN are RNs educated at the master's or post-master's level (DNP [doctor of nursing practice] or PhD [doctor of philosophy]) in a specific role and patient population. APRNs are prepared, by education and certification, to assess, diagnose, and manage patient problems, to order diagnostic tests, and to prescribe medications. ${ }^{23}$ APRNs include certified nurse practitioners, clinical nurse specialists, certified registered nurse anesthetists, and certified nurse-midwives. State licensing laws define the permissible scope of practice for RNs, as promulgated by state Boards of Nursing. All states regulate advanced practice nurses in some manner. Some license nurse practitioners; some grant authority to practice through certificates, recognition, or registration. In granting authority to practice beyond the RN's scope of practice, boards rely upon conventional authority mechanisms. These include graduation from approved educational programs and certification examinations. Many states rely on national certification programs to measure competency. APRNs typically are educated at the graduate level with in-depth preparation for a specialty practice, then take a certification exam, then comply with individual state licensing requirements. The National Council of State Boards of Nursing's " ${ }^{24}$ "Consensus Model for APRN Regulation: Licensure, Accreditation, Certification and Education" provides guidance for states to adopt uniformity in the regulation of APRN roles. However, considerable variation remains from state to state. Under the consensus model, all APRNs must pass a national certification exam. APRN practice is seen as building on the competencies of RNs, demonstrating a greater depth and breadth of knowledge, greater synthesis of data, increased complexity of skills and interventions, and having greater role autonomy.

APRNs have found jobs in acute care hospitals, managing care for specialty populations. They are also embedded in primary care as primary care providers. For example, rural health clinics in Iowa rely on senior clinical personnel such as physicians, physician assistants, and nurse practitioners to provide care coordination, care and case management, and identification of high-risk patients. ${ }^{25}$ APRNs also are seeing a renaissance in other expanded roles. For example, unit-based APRNs working in collaboration with a physician-hospitalist to manage patients on a general medicine unit in an academic medical center was 
the focus of a new unit-based role for APRNs at Vanderbilt University Medical Center. ${ }^{26}$ They noted that using APRNs in this way provided an opportunity for advanced practice nursing to assume a leadership role. Team effectiveness was a major focus. There has been considerable research done to compare patient outcomes of care provided by APRNs and physicians. The conclusion is that outcomes of care by APRNs in collaboration with physicians are comparable, and in some instances better, than care by a physician alone. A systematic review concluded that APRN care is safe, costeffective, and results in similar clinical outcomes and patient satisfaction as compared to care by physicians alone for the populations and in the settings of the reviewed studies. ${ }^{27}$ According to M Lofgren (personal communication, March $15,2014)$, APRNs are in great need of business skills for collaboration and role effectiveness in organizations. That being said, intentional leadership development will be required for current and future APRNs (Figure 1).

\section{Clinical leadership opportunities}

Health care systems and organizations must constantly gauge environmental forces and trends in patient care delivery to determine competency gaps within the workforce. Strategic and intentional development of clinical leaders can occur through education and training. The evidence base from business management research proves that leadership knowledge, skill, and abilities can be taught. Now it is clear that they should be taught in health care. The AACN's BSN (Bachelor of Science in Nursing), CNL, and DNP Essentials all require this. The Agency for Healthcare Research and Quality's (AHRQ) TeamSTEPPS initiative is an example of ways to address common clinical leadership challenges such as creating and maintaining high-functioning interdisciplinary teams. Thus, intentional leadership development is an important opportunity. Wilmoth and Shapiro ${ }^{28}$ have called for the adoption of a common framework for intentional leadership development that will enable nurses to lead at any level in any health care organization. Intentional leadership development can be conceptualized as new curricula, additional education, enabling innovation, use of reflective narratives about leadership roles, engaging in research on interdependency, and trialing of thinking strategies (Figure 1). This call is in synchronicity with national initiatives such as the AACN's DNP competencies, ${ }^{29}$ as seen in AACN Essential \#2 Organizations and Systems Leadership for Quality Improvement and Systems Thinking, and many professional nursing organizations. Intentional leadership development needs to be designed based on perceived educational needs and practice gaps to enable clinical leadership development at the point of care.

\section{Core concepts for clinical leadership development and education}

It is exciting to see new roles emerge for nurses as the US health care delivery system reconfigures to address cost, quality, and access issues. Nursing has been a profession rich in opportunities to grow and enrich the delivery of patient care services. Nurses find many practice settings and sites need the unique skills of an RN. The emergence of care coordination, CNL, and APRN roles has occurred in part because of the need for continuity of care and management of care transitions that were not well addressed in an acute care focused, episodic delivery system. Care was disconnected, chronic diseases were not well managed, and patients experienced gaps when only acute medical episodes were the focus. Care coordination has been demonstrated to be an important solution, common to all three roles. However, there are challenges to enabling creativity, problem solving, and innovation at the clinical leadership level for care coordination. These challenges arise in part because of the nature of knowledge acquisition in sciencebased professions, training in psychomotor skills that focus on repetitive task learning, and the use of structured clinical practice protocols, all of which tend not to expand and enable creativity and innovation.

As health care professions focus on their ever-expanding discipline-specific knowledge base, there is less time to teach and learn interdependence-based skills and have creative think time. The need to reduce variation through evidence-based practice and standardized protocols and the countervailing need to rapidly solve complex practice issues, such as when a serious event occurs like a fatal medication error due to a flaw in the process or poor communication, will cause a dynamic tension at the point of care. With a complex and rapidly changing health care environment, clearly the interconnection of evidence-based practice with ingenuity is essential to address and solve clinical practice problems, especially within multidisciplinary teams. Innovation and interdependency are two core concepts that serve as an opportunity to better inform clinical leadership development, education, and practice.

\section{Innovation}

Innovation is defined as use of a new mindset in a different context to enable creative linkages that will generate a solution or adaptation to a practice problem. Innovation requires that there be a wrap-around support system or environment 
that incubates innovativeness. The leader's role is to create supportive environments for inquiry, new mindsets, and contexts to foster innovation. ${ }^{30}$ This requires a differently developed set of clinical leadership competencies. Solutions for the practice environment are evolving toward evidencebased practice as the standard. Evidence-based practice, defined as unifying research evidence with clinical expertise and patient values and preferences, ${ }^{31}$ is being adopted by nurses and used as a marker of excellence. However, when implementing evidence-based practice, contexts will differ and best solutions may need to arise from innovation. This will require that leaders use a new mindset to successfully adapt recommendations for implementation. For example, under VBP, prevention of readmissions is an imperative. Thus the implementation of enhanced discharge planning is an evidence-based response. However, contexts differ, such as the degree to which electronic health records systems are compatible with each other and across settings and are adapted to discharge communication effectiveness. If poorly implemented, crucial medication administration information, such as discontinuing a medication when going home, may be lost between acute care and home care systems. The potential for catastrophic outcomes is significant. Clinical leadership is needed to generate innovative solutions. Another example is the use of a smartphone application for weight loss in overweight primary care patients. ${ }^{32}$ This shows how health care is changing with regard to how patient care issues are managed using home management augmented by technology.

Innovation enriches clinical leadership development and training because it enlarges the nurse's repertoire of strategies for problem solving in complex situations. As new issues emerge, creative solutions are needed. There may not be enough time to use incremental change strategies, such as in situations of sentinel events or serious safety near misses. If there is adequate time, the solution still may be complex. For example, technology solutions to access issues include implementation of telehealth. Opening a telehealth clinic at an employer's work site offers better access, but also involves complex infrastructure (secure video feed), equipment (telehealth carts with monitors, cameras, and diagnostic equipment), and staff (new roles for RNs and APRNs).

Use of strategies to enable creativity and problem solving will be critical (Huber et al, unpublished data, 2015). According to Berrett, ${ }^{33}$ there are various types of thinking approaches to enable problem solving for creativity and innovation. The key to problem solving is making unique connections. He discussed two thinking concepts that, if used intentionally, may support clinical leadership development. These include divergent thinking, which allows the clinical leader to come up with alternative ideas and theories to solve problems (generating creative ideas by exploring many possible solutions), and abductive logic, which relies on inference when information is incomplete (a medical diagnosis is an example). These two forms of scientific reasoning offer the clinical leader a novel way to reflect on and analyze problems, situations, and the context for implementation of strategies to achieve outcomes. Use of expanded thinking allows the individual to yield a large number of possible answers and make creative leaps in situations.

Developing a workplace context to support and enable a mindset for innovation will be essential. According to Joseph, ${ }^{30}$ a culture and climate for innovativeness is needed to spark and sustain the inquiry needed for innovation. Seven organizational antecedents are required to enable a culture and climate, so workers can innovate. These include organizational identification, organizational support, organizational values, relational leadership, nursenurse relationships, and nurse-nurse leader relationships. These findings illustrate that the work environment must be conducive to enabling innovation as an important element to reduce serious safety and quality issues and workforce shortages in health care. The author presented unit-level, interdepartmental-level, and system-level actions for all levels of leadership to enable innovation. When change is considered as either incremental change or revolutionary disruptive change, innovation is targeted at revolutionary disruptive change. Disruptive change is often needed for successful use of clinical leadership when multiple disciplines are interdependent or during rapid change in complex situations. This is because of the inertia or resistance to change commonly encountered when making planned changes in practice. Intentional leadership development in innovation is a clinical leadership opportunity.

\section{Interdependency}

Interdependency is defined as the extent to which each worker's performance and patient outcomes are dependent, controlled, or determined by other workers (Joseph et al, unpublished data, 2015). Interdependency enriches clinical leadership development and training because it focuses attention on the basic fact that, just like General Systems Theory pointed out how a change in one part of a system affects all parts of a system, so too do the actions of any one person affect all the others in the care team. Interdependency is a common phenomenon in clinical leadership roles of care coordination, $\mathrm{CNL}$, and $\mathrm{APRN}$ because care 
is complex, often delivered by a team, and performed across a continuum of care. Interdependency is intricately intertwined with teamwork and collaboration.

According to Stichler, ${ }^{34}$ recognition and acknowledgment of interdependence is critical to the development of a collaborative relationship. All members of the relationship must acknowledge that neither they nor any other person in the group can independently solve the problem or accomplish the stated goal, and they must have a willingness to voluntarily engage in the process. Collaboration is an interactive process characterized by mutuality, reciprocity, and interdependence that often leads to outcomes that could not be achieved otherwise. Every person in the interaction is affected by and affects the process of communication, creating a synthesis of cognitions, feelings, and expertise, which moves the internal system forward as a collective whole, rather than the sum of its collective parts. The work of Gerteis et $\mathrm{al}^{35}$ on patient-centered care demonstrated that interactions between providers, systems, and institutions affect patients' experiences both positively and negatively. For example, a competency for the CNL role is to participate as a team member and lead a team in the microsystem. Significant impact can be achieved through interdisciplinary teams charged with improving clinical outcomes, especially when the scope is between departments or locations of care. ${ }^{6}$

In complex care environments, interdisciplinary teams are used to address quality, safety, and effectiveness issues. There is a great need to enhance team formation and functioning in health care. For example, AHRQ of the US Department of Health and Human Services ${ }^{36}$ in collaboration with the Department of Defense's Patient Safety Program developed an evidence-based teamwork system designed for health care professionals as a solution to improving patient safety by improving communication and teamwork skills called TeamSTEPPS. AHRQ offers on its website readyto-use materials and a training curriculum to successfully integrate teamwork principles into all areas of a health care system. Using more than 20 years of research and lessons from the application of teamwork principles, many units or microsystems of organizations have done TeamSTEPPS training to address interdependency issues and create safety or quality improvement outcomes. ${ }^{37}$

\section{Recommendations for future clinical leadership development and education for nurses}

Patient care is increasingly moving to outpatient, community, and primary care settings in the US, and more nursing services are transitioning to community, outpatient, and home settings. ${ }^{38}$ This has created a new configuration for the practice of nursing that nurses have not been specifically prepared to fill in a care delivery system that traditionally was predominated by jobs in the acute care sector. Educators are challenged to develop, train, and educate nurses both for nursing practice in general and also for the types of jobs that nurses are projected to obtain. This is a complicated situation, given that it takes 4 years to prepare an $\mathrm{RN}$ at the baccalaureate level. Thus the vision for education needs to be at least 4 years out. The knowledge, skills, and abilities for clinical leadership can and should be taught at the BSN level in order to prepare students for clinical leadership roles. Clinical leadership roles occur postlicensure, with education being postbaccalaureate at the Master's (CNL) or DNP level. Education and training is based on a combination of specific experience levels and further knowledge acquisition. Thus, clinical leadership requires additional education and experience. Because changing a curriculum does not produce an immediate change in the types of graduates or their preparation for specific care environments, shifting the preparation of nurses for expanded settings and sites with reconfigured care integration roles will take time, effort, leadership development strategies in practice, and strategic curriculum transformations.

Since clinical leadership requires additional education and experience, engaging in practice narratives or stories can enable ongoing clinical leadership development. Smith and Liehr's ${ }^{39}$ middle-range theory of attentively embracing story can be used as a structure to guide reflective leadership development. This theory consists of prompts for reflection that offer a window to look inside the self and to know one's self as one strives toward understanding and realizing the meaning of desirable or best practice. The use of storytelling or documentation of narratives is recounting one's current situation to clarify present meaning in relation to the past with an eye to the future. Clinical leaders need to reflect on three prompts: 1) how did I feel in this situation?; 2) what were the core tasks or interactions used in this situation?; and 3) how do I ensure self-development and support in a similar situation? This process of reflection will provide insight into future behavior in a similar situation or new situation. It is clear that nurses need to be life-long learners, using development, training, reflection prompts, and advanced education to acquire specialized knowledge and skills.

Berrett $^{33}$ questioned how society would compete in a global economy if students are being taught how to adapt 
to yesterday's world in today's world. He recommended that creativity should be infused in all curricula. That being said, identifying innovations for care coordination, the CNL, and APRN roles will require crossing boundaries to become exposed to new contexts to innovate ${ }^{40}$ (Joseph et al, unpublished data, 2015). Joseph et al (unpublished data, 2015) have described a curriculum innovation designed to have students observe coordination and collaboration in other industries to apply a new mindset and examine the situation in new contexts to determine linkages for solutions in health care or nursing practice.

Interdependency needs to be studied and understood beyond the concept of collaboration to ensure role effectiveness and clinical outcomes. The goal is high-functioning teams that respond rapidly and create and innovate effective solutions to practice problems, thus demonstrating clinical leadership. There is a growing evidence base to draw from. For example, in a 2009 study, Joseph et $\mathrm{al}^{41}$ developed a six-item interdependency scale based on a previous conceptual framework of interdependence structure of interpersonal situations by Rusbult and Van Lange. ${ }^{42}$ The Cronbach $\alpha$ for the study scale was 0.687 from a sample of 317 unique observations in a 1,200-bed tertiary hospital in the Southern United States. This measure has promise for enabling awareness, responsibility, and leadership within teams to affect patient outcomes. The scale allowed team members to examine their level of interdependency in the context of the team and examine whether team members understand each other's roles in the context of achieving patient goals and outcomes.

\section{Conclusion}

Nursing practice is at a cusp where leadership competencies are imperative to ensure the reconfiguration of health care delivery in support of the ACA and health care delivery. The future of health care warrants new roles and ways of leading to ensure solutions for a changing environment. The call for the adoption of a common framework for intentional leadership development by Wilmoth and Shapiro ${ }^{28}$ is timely for care coordinators, CNLs, and APRNs in an evolving health care environment (Figure 1). A great way to start is by using available resources for the development and education of nurses in these essential clinical leadership roles. Two major concepts at the core of these evolving roles are innovation and interdependency. Curriculum, in-service training, narrative, thinking strategies and reflective practice, continuing education, and other educational efforts can target these concepts as a way of augmenting knowledge, skills, and abilities in care coordination and clinical leadership in nursing.

\section{Disclosure}

The authors report no conflicts of interest in this work.

\section{References}

1. Joynt J, Kimball B. Innovative Care Delivery Models: Identifying New Models that Effectively Leverage Nurses. Princeton, NJ: Robert Wood Johnson Foundation; 2008.

2. Smolowitz J, Speakman E, Wojnar D, et al. Role of the registered nurse in primary health care: meeting health care needs in the 21 st century. Nurs Outlook. 2015;63(2):130-136.

3. Institute of Medicine. The Future of Nursing: Leading Change, Advancing Health. Washington, DC: National Academies Press; 2010.

4. Huber DL. Leadership and management principles. In: Huber DL, editor. Leadership and Nursing Care Management. 5th ed. St Louis, MO: Elsevier; 2014:1-36.

5. McCausland MP. Opportunities and strategies in contemporary health system executive leadership. Nurs Adm Q. 2012;36(4):306-313.

6. Thomas PL, Roussel L. Clinical nurse leadership: creating the vision. In: Harris JL, Roussel L, Thomas PL, editors. Initiating and Sustaining the Clinical Nurse Leader Role: A Practical Guide. Burlington, MA: Jones \& Bartlett Learning; 2014:481-500.

7. Wong CA, Cumming, GG, Ducharme L. The relationship between nursing leadership and patient outcomes: a systematic review update. J Nurs Manag. 2013;21(5):709-724.

8. Cummings GG, MacGregor T, Davely M, et al. Leadership styles and outcome patterns for the nursing workforce and work environment: a systematic review. Int J Nurs Stud. 2010;47(3):363-385.

9. Clavelle JT, Drenkard K, Tullai-McGuinness S, Fitzpatrick JJ. Transformational leadership practices of chief nursing officers in Magnet $^{\circledR}$ organizations. J Nurs Adm. 2012;42(Suppl 10):S3-S9.

10. American Organization of Nurse Executives (AONE). Nurse executive competencies. Nurse Leader. 2005;3(1):15-21.

11. Kelly LA, Wicker TL, Gerkin RD. The relationship of training and education to leadership practices in frontline nurse leaders. J Nurs Adm. 2014;44(3):158-163.

12. Tourangeau A, McGilton K. Measuring leadership practices of nurses using the leadership practice inventory. Nurs Res. 2004; 53(3):182-189.

13. Jamison M, Ross VM, Hornberger CA, Morse VL. Implementation of the care coordinator role: a grounded theory approach. J Prof Nurs. 1999;15(6):356-363.

14. Huber DL. Case and population health management. In: Huber DL, editor. Leadership and Nursing Care Management. 5th ed. St Louis, MO: Elsevier; 2014:197-225.

15. Powell SK, Tahan HA. CMSA Core Curriculum for Case Management. 2nd ed. Philadelphia, PA: Lippincott Williams \& Wilkins; 2008.

16. Commission for Case Manager Certification (CCMC). NEW INFOGRAPHICS: CMCoordinates for Role and Function. Available from: http:/ccmcertification.org/center-stage/new-infographicscmcoordinates-role-function-0. Accessed February 5, 2015.

17. Commission for Case Manager Certification (CCMC). Certification Guide to the $C C M^{\circledR}$ Examination. Mount Laurel, NJ: CCMC; 2015

18. California Institute for Nursing and Healthcare. Nurse Role Exploration Project: The Affordable Care Act and New Nursing Roles. 2013. Available from: http://www.caactioncoalition.org/cinhc-releases-nurserole-exploration-project-report. Accessed April 23, 2015.

19. American Association of Colleges of Nursing (AACN). White Paper on the Role of the Clinical Nurse Leader. Washington, DC: AACN; 2007.

20. Harper DC. Foreword. In: Harris JL, Roussel L, Thomas PL, editors. Initiating and Sustaining the Clinical Nurse Leader Role: A Practical Guide. Burlington, MA: Jones \& Bartlett Learning; 2014:xiii-xiv.

21. American Association of Colleges of Nursing (AACN). Competencies and Curricular Expectations for Clinical Nurse Leader Education and Practice. Washington, DC: AACN; 2013. 
22. Binder M. The current evidence base for the clinical nurse leader: a narrative review of the literature. J Prof Nurs. 2014;30(2):110-123.

23. National Council of State Boards of Nursing. APRNS in the US. 2015. Available from: https://www.ncsbn.org/aprn.htm. Accessed May 23, 2015.

24. National Council of State Boards of Nursing. Consensus Model for APRN Regulation, Licensure, Accreditation, Certification and Education. 2008. Available from: https://www.ncsbn.org/736.htm. Accessed April 23, 2015.

25. Wright B, Damiano PC, Bentler SE. Implementation of the affordable care act and rural health clinic capacity in Iowa. J Prim Care Community Health. 2015;6(1):61-65.

26. Feistritzer NR, Jones PO. A proof-of-concept implementation of a unit-based advanced practice registered nurse (APRN) role. Nurs Clin North Am. 2014;49(1):1-13.

27. Newhouse R, Stanik-Hutt J, White KM, et al. Advanced practice nurse outcomes: 1990-2008: a systematic review. Nurs Econ. 2011 ;29(5):1-21.

28. Wilmoth MC, Shapiro SE. The intentional development of nurses as leaders: a proposed framework. J Nurs Adm. 2014;44(6):333-338.

29. American Association of Colleges of Nursing (AACN). The Essentials of Doctoral Education for Advanced Nursing Practice. Washington, DC: AACN; 2006.

30. Joseph ML. Organizational culture and climate for promoting innovativeness. J Nurs Adm. 2015;45(3):172-178.

31. Stevens K. The impact of evidence-based practice in nursing and the next big ideas. Online J Issues Nurs. 2013;18(2):4.

32. Laing BY, Mangione CM, Tseng C, et al. Effectiveness of a smartphone application for weight loss compared with usual care in overweight primary care patients: a randomized, controlled trial. Ann Intern Med. 2014;161(10):S5-S12.
33. Berrett D. Creativity: a core for the common curriculum. Chron High Educ. 2013;79(2):13-20.

34. Stichler J. Professional interdependence: the art of collaboration. $A d v$ Pract Nurs Q. 1995;1(1):53-61.

35. Gerteis M, Edgman-Levitan S, Daley J, Delbanco TL. Introduction: medicine and health from the patient's perspective. In: Gerteis M, Edgman-Levitan S, Daley J, Delbanco TL, editors. Through the Patient's Eyes: Understanding and Promoting Patient-Centered Care. San Francisco, CA: Jossey-Bass Publishers; 1993.

36. Agency for Healthcare Research and Quality (AHRQ). About TeamSTEPPS. Rockville, MD: AHRQ; 2015.

37. Ward MM, Zhu X, Lampman M, Stewart GL. TeamSTEPPS implementation in community hospitals. Int J Health Care Qual Assur. 2015; 28(3):234-244.

38. Orr P, Davenport D. Embracing change. Nurs Clin North Am. 2015; 50(1):1-18.

39. Smith MJ, Liehr P. The Theory of Attentively Embracing Story. Middle Range Theory in Nursing. New York, NY: Springer Publishing; 2003.

40. Isaacson W. The Innovators: How a Group of Hackers, Geniuses, and Geeks Created the Digital Revolution. New York, NY: Simon \& Schuster; 2014.

41. Joseph L, Bogue R, Zhu X. Interdependency of clinical roles for patientcentered care. Presented at: International Conference on Communication in Healthcare, Oct 2009, Miami Beach, FL.

42. Rusbult CE, Van Lange PA. Interdependence, interaction, and relationships. Annu Rev Psychol. 2003;54:351-375.
Journal of Healthcare Leadership

\section{Publish your work in this journal}

The Journal of Healthcare Leadership is an international, peer-reviewed, open access journal focusing on leadership for the health profession. The journal is committed to the rapid publication of research focusing on but not limited to: Healthcare policy and law; Theoretical and practical aspects healthcare delivery; Interactions between healthcare and society and evidence-based practices;

\section{Dovepress}

Interdisciplinary decision-making; Philosophical and ethical issues; Hazard management; Research and opinion for health leadership; Leadership assessment. The manuscript management system is completely online and includes a very quick and fair peer-review system. Visit http://www.dovepress.com/ testimonials.php to read real quotes from published authors. 